\title{
Emergency and post-emergency control in the formation of micro-grids
}

\author{
Pavel Ilyushin ${ }^{1 *}$ \\ ${ }^{1}$ Petersburg power engineering institute of professional development, Aviation str. 23, Saint Petersburg, Russia
}

\begin{abstract}
At the present time, there is a worldwide tendency to clustering the electrical power systems at LV and MV levels - creating the micro-grids. In this connection, numerous of technical issues appear in control approaches in general and in particular in emergency and post-emergency control. As micro-grids are meant to operate both in interconnected and islanded modes, the ability to steadily island and backward synchronize with an upper grid provides reduction of the negative impact of failure rate of electrical power system's elements, as well as power quality parameters influence toward operation regime of the loads. The consequence of previously described features is particular requirements for the following emergency automatics: overload-preventing automatics for overhead lines, cables and transformers; out-of-step automatics and under-frequency load shedding. Low inertia constants of some distributed generation units and low time constants of electromagnetic transients of generating units and ESS, interconnected with a grid by means of power-electronics, provide an ability to realize additional (fast) control signals in overloadpreventing automatics, dispatching generating units and ESS with an aim to prevent considered elements tripping.
\end{abstract}

\section{Introduction}

Generation capacity decentralization forced by distinctive factors, including evolving of economically justified low-power energy generation and storage technologies (installed capacity less than $670 \mathrm{~kW}$ ), added by electricity market liberalization, reflecting in price-responsive demand as a measure of consumer's involvement, end up with the formation of physical and virtual (consumption reduction) lump generation capacities in MV/LV distribution grids.

Being planned as passive one-port, distribution grids acquire steady-state regime control (steady-state stability) and transient control (transient stability) requirements, inherent for transmission networks, as cumulative install capacity of MV/LV generating units grows up.

Energy storage deployment is always coupled with automatic control system tying, which also can be used for implementation of demand response.

An aggregation of DG units, energy storage units and controllable loads, forming together so called DERs, by control integrity leads to the origin of controllable distribution grid's concept with the following objectives: - Reduction of uncontrollable influence of commissioned and future DG objects towards both steady-state and transient regime parameters,

- Creation of the technological basis of nondiscriminatory access for dispatched and non-dispatched «heterogeneous» DERs (comprising renewable energy sources),
- Creation of the technological basis for implementation of demand response,

- Creation of the technological basis for realization of ancillary services by DERs, which value in overall balance grows proportionally to the penetration level [1].

All abovementioned leads to a concept of micro-grid - an aggregation of electrically tied loads and DERs, having fixed electrical boundaries and operating as a single controllable element in both synchronized with an external grid and islanded modes.

In general, the appearing of this concept pursues economically effective local load supply. According to [2], micro-grid's electrical boundaries are the follows:

- A part of distribution grid limited by mains' circuit breaker (CB) (MV or LV),

- LV/MV feeder,

- Consumer's internal-supply electrical grid.

\section{Emergency regimes in islanded micro- grids}

Emergency regimes in islanded micro-grids are generally characterized by heavier burden from consumer's perspective than those under synchronized conditions, especially when accompanied by power deficit. Planning of emergency control encompassing electrical power supply normalization under micro-grid's islanded modes with part of generation cut off, load rise and etc. should be allocated as distinguished task due to the following:

Corresponding author: ilyushin.pv@mail.ru 
1. The field of those solutions is much wider in comparison with autonomous electric power supply systems. The likelihood of micro-grids, synchronized with the mains, to be islanded is inevitable, especially in case of equipment tripping in external grid under maintenance conditions (N-2). The problem generally worsens with deterioration of distribution grid's equipment, recruitment of skill-less manpower, related to emergency automatics (EA), and insufficient redundancy level.

2. Loss of a generator, a group of generators or an entire power plant cannot lead to significant active power deficit and another-reason supply interruption (voltage reduction and etc.) in an interconnected grid, whereas the same tripping events can cause full outage of reliable loads in islanded mode. Depending on microgrid's islanding scenario, balance of generation and consumption can bias in diametrically opposite ways, such that it leads either to necessity of shedding a part of generating units or to $100 \%$ power deficit. Evidently, all the preceding should be considered while planning EA.

3. Technical features of modern generating units, such as gas turbines, reciprocating engines, wind energy conversion systems, photovoltaics, drastically differs from those of conventional steam turbines. Mainly, it relates to parameters, defining transient dynamics under external disturbances.

4. There is a tendency toward technical and economical characteristics enhancement of generating units, manufacturing abroad, which is on the strength of tripping times reduction and, thereafter, fastening of normal operating condition restoration due to applying of microprocessor-based protection.

Consequently, both islanding realization and ensuring continuous sustainable power supply in islanded mode should be evaluated while planning micro-grids, taking into account the aspects listed below:

- Frequency and active power control of generating units,

- Adjustments of generator voltage threshold $\mathrm{U}_{\mathrm{r} 0}$,

- Load transient-stability margins influence toward EA planning,

- Fault-voltage ride through characteristics of a microgrid,

- Load shedding arrangement under unintentional islanding of a micro-grid,

- Out-of-step protection for generating units,

- Usage of energy storage devices.

\section{Frequency and active power control}

Commonly, generating units, operating in parallel with mains, acquire constant active power control, in autonomous grids - constant frequency control, whereas those tended to operate in both - combined control with switching between in the moment of islanding and synchronization respectively. The cornerstone here is an algorithm used as it can function incorrectly.

As a widespread practice, preselected CB's states (up to 5 depending on manufacturer) are used as flags for switching. Newer-the-less, in exploitation islanding can occur in neither of those $\mathrm{CBs}$, non-causing algorithm to switch, resulting in frequency oscillations with possible violation of generating unit's frequency margins, ending up with this generating unit cut off.

Identical scenario can take place also when active power change is taken as the threshold. While generating units track fixed active power step, an islanding can be characterized by lower absolute value or an opposite sign.

The reasons of micro-grids to island comprise emergency equipment tripping, especially in maintenance schemes and due to EA trips, where capacity of remaining connected loads may vary drastically. If islanding happens with high load burden, load shedding takes place.

One of the load shedding algorithms applied on gasturbine units is considered below. The latter adopts $50 \%$ or higher power loss during $200 \mathrm{~ms}$ as a threshold to reveal an islanding. It is assumed that generating unit has $80 \%$ active power in parallel mode. In calculations below 7 scenarios different in load value are depicted. Load varies from 5 to $100 \%$ of generating unit nominal power. Regime parameters, worth to be highlighted under question, are active power and frequency. The main calculation parameters:

- Active power control in synchronized regime: regulation actions keeping $\mathrm{Pg}=$ const,

- Kotating speed control: 0,5\% droop with power increase and decrease time constants of $800 \mathrm{~ms}$ and 650 ms respectively,

- $60 \%$ of asynchronous rotating load.

Calculation results reveal small dependence on whether external fault is incepted in previous-toislanding mode or not. Fig. 1 depicts frequency and active power curves for generating unit with constant active power for various load steps under fault and normal conditions respectively. It can be seen that differences take place only at initial stage of approximately two times fault duration.

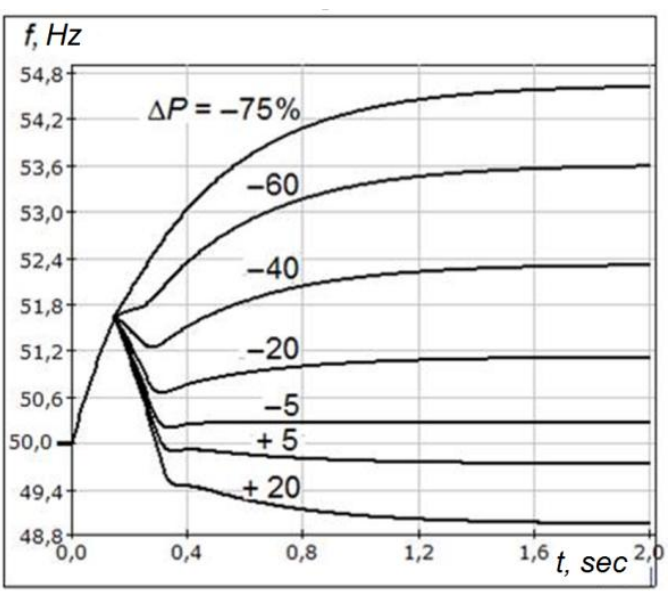

a) 


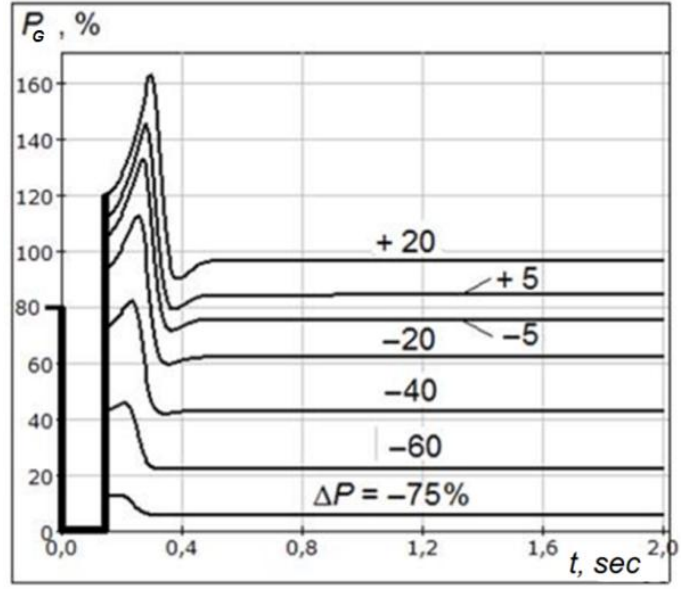

b)

Fig. 1. Frequency (a) and active power (b) under islanding conditions for no turbine fuel control and different load steps $\Delta \mathrm{P}$ under three-phase short-circuit incepted in previous-toislanding regime.

For load steps of $60 \%$ and $75 \%$ switching to rotating speed (frequency) control takes place, otherwise, generator continuously regulates its output active power. So, as load drops less than $50 \%(\Delta \mathrm{P}$ of -40 to $-5 \%$ in Fig. 1) with respective frequency rise, the regulator approaches the preset power value, feeding more fuel to combustor, which provokes further frequency rise. Load surges $(\Delta \mathrm{P}$ of $+5,+20 \%$ in Fig. 1$)$ cause increment in frequency reduction.

Another principle, briefly mentioned above, consist in watching chosen CB's states. Whether a part of them are on, a regime is defined as parallel with mains, otherwise - islanded. This paradigm does not consider scenarios of grid splitting at different CBs, as shown in Fig. 2.

Based on preceding, the following findings are made:

1. The value of load steps cannot be used as a threshold, as it is strictly defined by previous-toislanding regime: various in absolute value and sign load steps in various topological and parametrical situations.

2. Error penalization should be considered for combined regulators, as continuation to operate in power control mode in case of micro-grid islanding likely to lead to power outage, whereas, only inaccuracy of power dispatch occur in the opposite situation, when gas turbines operate with frequency droop in parallel regime.

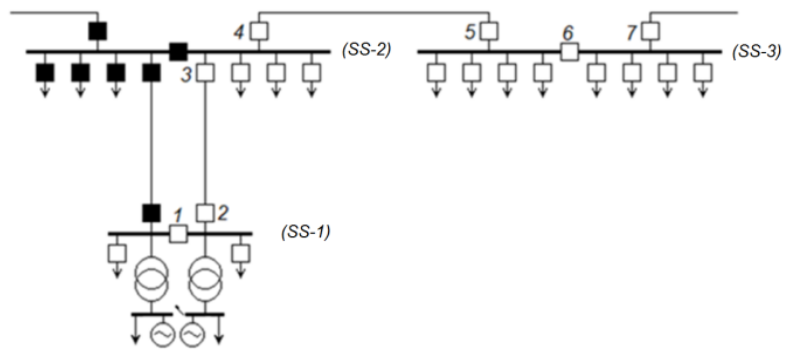

Fig. 2. For one busbar shutted down for maintenance tripping of CB 1-7 (3-7 are uncontrollable) will lead to mains loss.
A gas turbine with frequency droop of $4-5 \%$ without secondary control has power error of up to $5 \%$ nominal power for $0,1 \mathrm{~Hz}$ frequency oscillations.

As a solution for combined regulation issues, continuous frequency droop of 4-5\% (Fig. 3) should be applied irrespectively. It can easily be done for lowpower generating units by simply blocking the switching algorithm and setting the droop of $4-5 \%$.

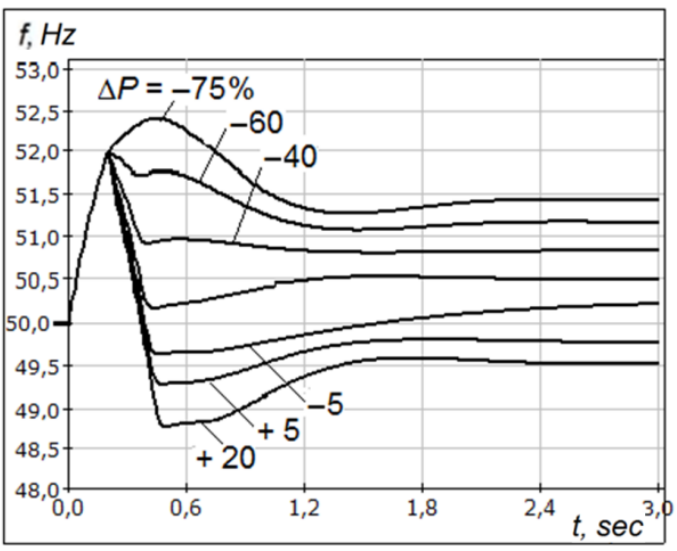

Fig. 3. All the above processes but with continuous speed (frequency) regulation.

\section{Generator voltage threshold adjustments}

For low generator voltage $\mathrm{U}_{\mathrm{G} 0}$ threshold values reactive power is chiefly drawn from an external grid. So, in case of islanding $10 \mathrm{kV}$ busbar voltage drops $(\Delta \mathrm{U})$, biasing the voltage-regime point to up to voltage collapse. It alleviates active power deficit, where even frequency surge is possible. Fig. 4 depicts pre-fault generator Q value correlation with voltage $\Delta \mathrm{U}$ and frequency at initial stage of islanded mode. Those parameters are expressed as generator voltage function in pre-fault regime with no under-frequency load shedding considered.

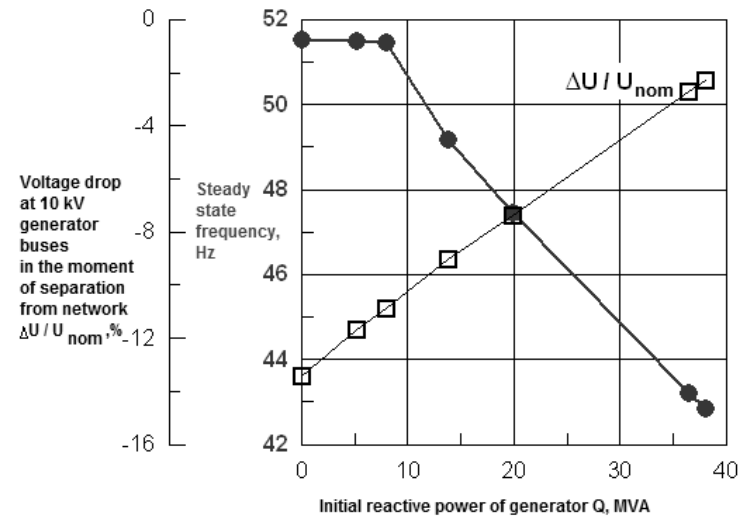

Fig. 4. The influence of generator voltage preset value toward islanding success.

If generator covers entire reactive load, the voltage drop is minimal (lower than $4 \%$ in absolute value); the loads are within their transient stability margins; the 
active power deficit is reflected in frequency reduction (Fig. 5a).

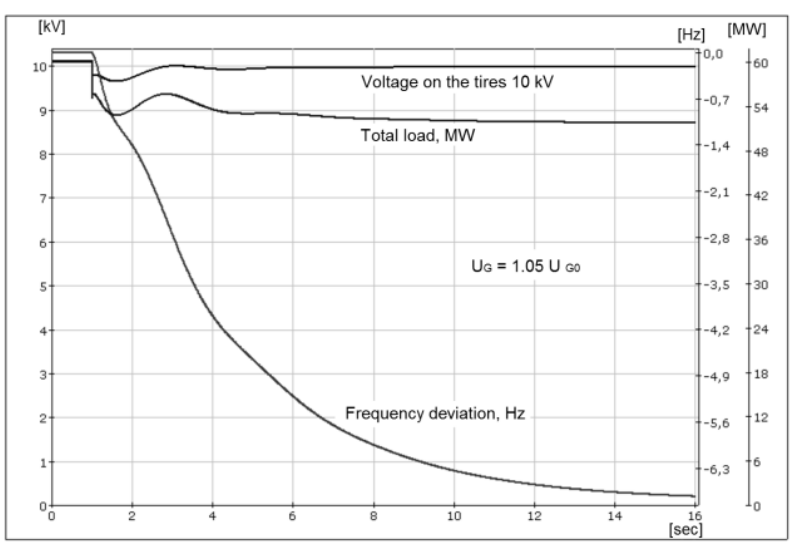

a)

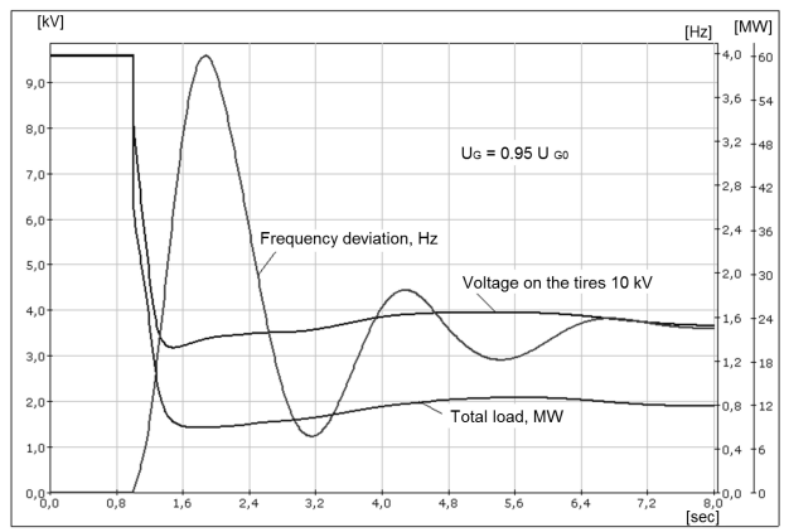

b)

Fig. 5. Reactive power regimes: a - zero reactive power flow from mains, $b$ - non-zero reactive power flow from mains.

Quite opposite situation appears if generator excitation is relatively low. In pre-fault regime reactive power is taken from an external grid $(\mathrm{Q}<0)$. At the moment of islanding the voltage drop takes place as low as preset $\mathrm{U}_{\mathrm{G} 0}$ value. Asynchronous motors' slip rises, leading to further voltage reduction and, finally, voltage collapse. The process is shown in Fig. 5b, where load outage happens initially due to voltage drop, then - due to voltage collapse on the time span of $0,1 \mathrm{~s}$ in overall. The frequency point reaches its peak at $54 \mathrm{~Hz}$ with steady-state value of $51,5 \mathrm{~Hz}$. Eventually, all loads are inoperative, because of voltage value lower than $40 \%$ nominal. In the second case, voltage collapse and full outage take place.

Summing up, it is advisable to consider all the abovementioned processes while calculating $\mathrm{U}_{\mathrm{G} 0}$ threshold value.

\section{Load stability margins influence toward emergency automatics selection}

If islanding proceeds without load stability margins violation, lags in under-frequency load shedding (also, frequency thresholds lowering in comparison to maximum possible) do not cause influence toward post- emergency conditions, besides short-term frequency reduction is unacceptable for generators.

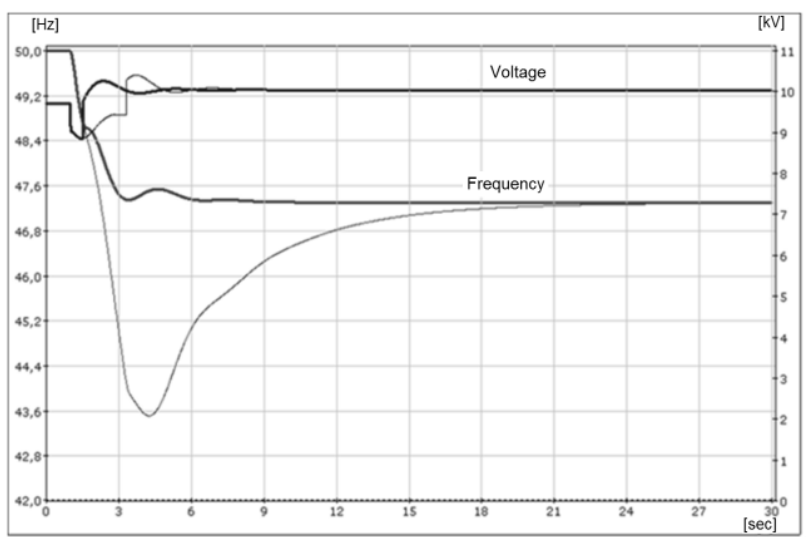

a)

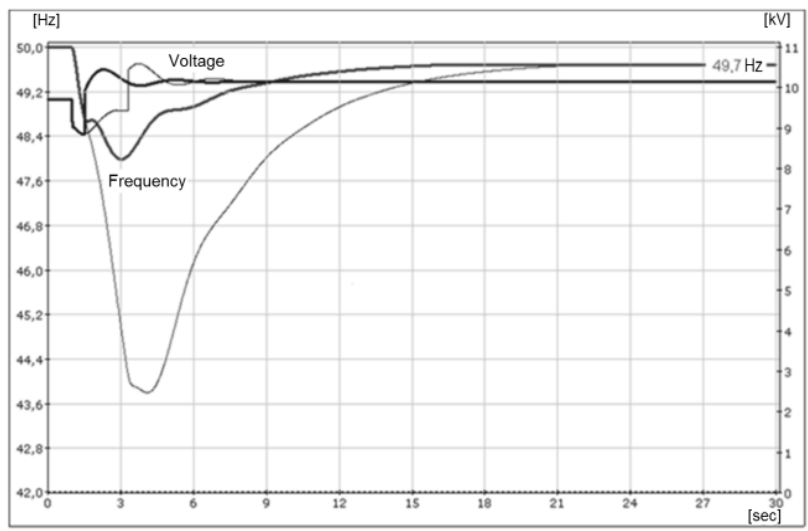

b)

Fig. 6. Islanding with initial active power deficit of $46 \%$. Under-frequency load shedding trips at $49,2 \mathrm{~Hz}$, the lag of load shedding are 0,2 s (bold curves) and 2,0 $\mathrm{s}$ (thin curves).

It is evident that lags do not affect the regime if transient frequency reduction satisfies relevant generator and load margins. Two processes distinctive in underfrequency load shedding volume and realization time are shown in Fig. 6 a), b). The assumptions made here are the following: shedding initialization at $49,2 \mathrm{~Hz}$ with 0,2 $\mathrm{s}$ lag for the first (bold) and 2,0 s for the second (thin), with $30 \%$ and $35 \%$ cut-off volume respectively.

Generally transient deep frequency reduction is allowed for vast majority of power receivers. Newer-theless, selection of EAs, its thresholds and stimulus' volume should be accompanied by load stability analysis.

\section{Islanding success under fault conditions}

If micro-grid islanding is preformed under fault conditions (for instance, three-phase short-circuit in proximity of substation busbar is taken), motor transient stability upset takes place with extensive load outage and consequent frequency rise. Fig. 7 depicts characteristic curves both with short-circuit of $0,2 \mathrm{~s}$ duration and without short-circuit. 


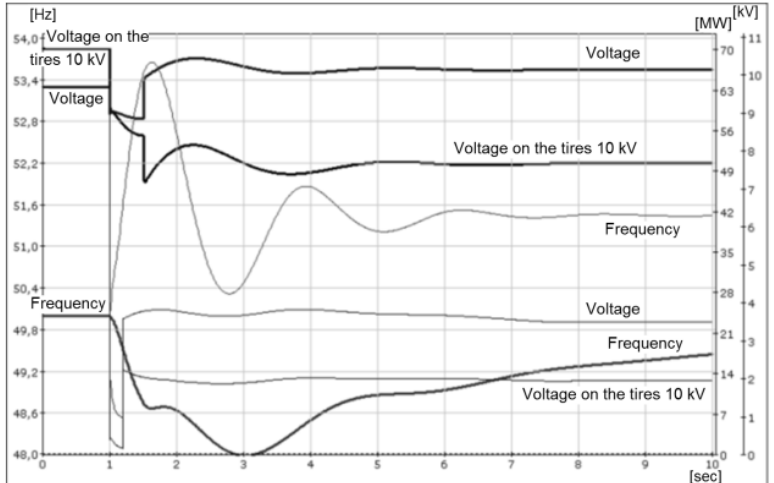

Fig. 7. Islanding under three-phase short-circuit conditions (thin curves) and without short-circuit (bold curves).

Drawn graphs depict about 30\% load drop, voltage rise up to allowed boundaries after insignificant voltage drop and frequency rise up to $51,5 \mathrm{~Hz}$, which in overall corresponds to successful islanding.

As a conclusion, short-circuit modeling should be conducted to assess the likelihood of successful islanding while planning micro-grids.

\section{Isalding followed load shedding}

Fast-operating load shedding is required in all of those cases characterized by significant voltage drop, load power drop and load transient stability upset.

As initial values for calculations the same cut-off volume (35\%) is taken, but initializations are performed in $0,1 \mathrm{~s}$ after short-circuit inception and $0,3 \mathrm{~s}$ after clearance. Fig. 8 reveal self-acceleration of rotating load after fast shedding.

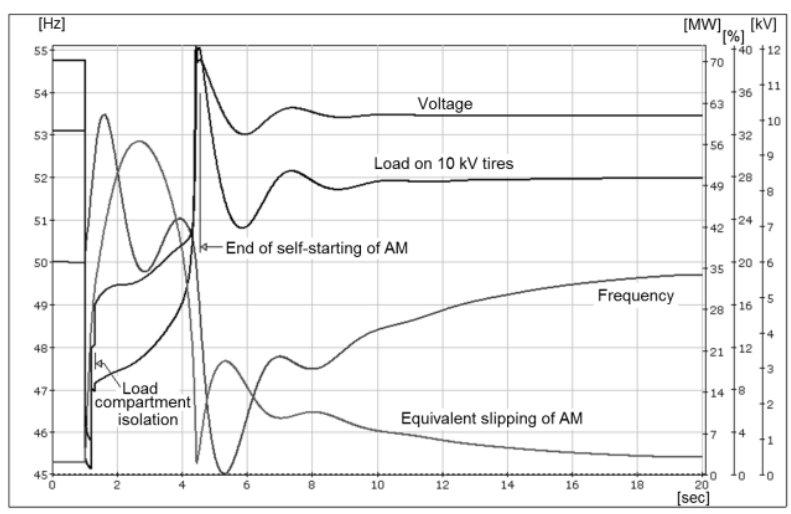

Fig. 8. Load shedding in $0,3 \mathrm{~s}$ after fault inception and 0,1 after clearance.

Implementation of load shedding, triggering in the moment of islanding, is justified if under-frequency load shedding operating speed is significant, this-type automatics failure due to deep voltage drops is inevitable (in most cases deep frequency reduction is accompanied by deep voltage decrease). Moreover, there is a probability to face lack in power available for shedding under high-power deficit conditions, which can end up with generation facilities' auxiliaries out of service.

\section{Out-of-step protection}

Inherent low inertia of reciprocating engines and two-/ three-shaft gas turbines (with free power turbine) possess considerable difficulties in transient stability ensuring of the abovementioned units. In addition, being the main reason of secondary dynamic stability upset, small electrical distances of MV/LV grids contribute to load stability loss in case of generator asynchronous regime takes place.

Fast clearance of generator asynchronous mode by its tripping off have the following consequences should be bear in mind while selecting out-of-step algorithm and corresponding thresholds:

- Microgrid-mains tie power flow increase in case of internal generation loss,

- The possibility of equipment overload (transformers, overhead lines) as vast majority of micro-grid loads is located at MV/LV levels,

- Voltage decrease at micro-grid nodes, where the most susceptible loads are synchronous and asynchronous motors of industrial facilities.

It is justified to allow asynchronous running of lowpower generators if asynchronous satisfies the following:

- Short duration,

- Ending up with resynchronization of the generators,

- Not causing violation of normal regime bounds of other generators and power receivers (should be confirmed by calculations),

- The duration is controlled by out-of-step protection.

Tripping generators off by out-of-step protection is justified if asynchronous mode duration exceeds an allowed value, which, generally, occurs in maintenance schemes and emergency regimes with "weak" ties. The likelihood of spontaneous resynchronization of generators is high in micro-grids, making adequate to offset out-of-step protection tripping if it does not lead to multifrequency asynchronous running of the generators and motor stability loss.

Described above issue can be fully solved by conducting transients modeling, taking into account load transient parameters in adjacent nodes. It also allows to depict impedance hodograph diagram used for out-ofstep protection parametrization.

\section{Energy storage usage}

Hereafter rapidly adjustable in active and reactive power energy storage is considered. The operating speed is assumed to be that high, so switching to controllable feeding/absorbing of active/reactive power within emergency regime is instantaneous in a first approximation.

The viability of energy storage devices is defined by economic factors. All expenses are counterbalanced and even outweighed for susceptible facilities.

Energy storages are applied within micro-grids under islanding mode in the following cases:

- Safety trips of power source, where energy storages are aimed to restore normal supply scheme and ensure failure-free manufacturing shutdown, 
- To cover local equipment overloads under extraordinary conditions (substantial weather changes, equipment emergency trips and ets.),

- Generator black-start source,

- Emergency source for reliable loads under autoreclosing and automatic transfer switch pauses,

- As a measure for voltage collapse tackling in grids with high share of rotating loads.

It worth to note, that voltage flattening out to prefault regime value cannot always be performed by energy storage effectively. For short-circuits in proximity of load terminals the energy storage power needed to lift voltage up to nominal can be significant. The fault clearance fastening can alleviate the situation.

Energy storage application within micro-grids provides several benefits, however the quantitative effect should always be assessed by calculations.

\section{Conclusion}

The analysis of successful islanding possibility and sustainable power supply in micro-grid island mode should always be conducted, taking into account all technical questions described in this paper.

It is justified to implement unified speed (frequency) control algorithm with frequency droop of $4-5 \%$, depending on prime mover, irrespectively to projected generator operating regime.

The deep under-excitation of micro-grid generators can lead to significant voltage reduction, motor's slip rise and further process progress up to voltage collapse. Thus, the generator voltage preset value should be set relatively high.

To prevent voltage collapse of a micro-grid, islanding under fault conditions, the fast-operating load shedding, tripping by a signal formed at the moment of islanding, should be implemented, as under-frequency load shedding can operate ineffective.

As micro-grid generators spontaneous resynchronization is very likely, offsetting out-of-step protection by time is justified in all cases, but if leading to multifrequency asynchronous running of the generators or load transient stability upset.

Application of energy storage devices within microgrids is economically effective for industrial facilities, characterized by high reliability requirements and financial losses for short-term power outages.

\section{References}

1. P. Ilyushin, S. Muzalev, Relay protection and automation 3, 39-45 (2016)

2. N. Hatziargyriou, Wiley-IEEE Press 1, 344 (2014)

3. P. Ilyushin, Electro 4, 19-25 (2011) 